\title{
Fear conditioned potentiation of the acoustic blink reflex in patients with cerebellar lesions
}

\author{
Matthias Maschke, Johannes Drepper, Kerstin Kindsvater, Florian P Kolb, \\ Hans-Christoph Diener, Dagmar Timmann
}

\begin{abstract}
Objective-To investigate whether the human cerebellum takes part in fear conditioned potentiation of the acoustic blink reflex.

Methods-A group of 10 cerebellar patients (eight patients with lesions involving the medial cerebellum, two patients with circumscribed lesions of the cerebellar hemispheres) was compared with a group of 16 age and sex matched healthy control subjects. The fear conditioned potentiation paradigm consisted of three phases. During the first, habituation phase subjects received 20 successive acoustic blink stimuli. In the subsequent fear conditioning phase, subjects passed through 20 paired presentations of the unconditioned fear stimulus (US; an electric shock) and the conditioned stimulus (CS; a light). Thereafter, subjects underwent the potentiation phase, which consisted of a pseudorandom order of 12 trials of the acoustic blink stimulus alone, 12 acoustic blink stimuli paired with the conditioned stimulus, and six conditioned stimuli paired with the unconditioned stimulus. The EMG of the acoustic blink reflex was recorded at the orbicularis oculi muscles. The potentiation effect was determined as the difference in normalised peak amplitude of the blink reflex evoked by pairs of CS and acoustic blink stimuli and evoked by the acoustic stimu-
\end{abstract} lus alone.

Results-In the habituation phase, short term habituation of the acoustic blink reflex was preserved in all cerebellar patients. However, in the potentiation phase, the potentiation effect of the blink reflex was significantly reduced in patients with medial cerebellar lesions compared with the controls (mean (SD) potentiation effect $(\%)$, patients: -6.4 (15.3), controls: 21.6 (35.6)), but was within normal limits in the two patients with lateral lesions.

Conclusions-The present findings suggest that the human medial cerebellum is involved in associative learning of nonspecific aversive reactions-that is, the fear conditioned potentiation of the acoustic blink reflex.

(F Neurol Neurosurg Psychiatry 2000;68:358-364)

Keywords: cerebellum; fear conditioned potentiation; acoustic blink reflex; associative learning
Several animal and human studies indicate that the intermediate cerebellum is involved in conditioning of specific aversive reactions, in particular of the eyeblink and limb flexion reflex. ${ }^{12}$ Animal studies suggest an additional role of medial parts of the cerebellum in habituation and conditioning of non-specific aversive reactions. For example, the cerebellar vermis has been shown to have a role in long term habituation of the acoustic startle reflex. ${ }^{3-5}$

The startle reflex is a non-specific aversive reaction which preserves animals from blows or attacks by stiffening the muscles of neck, body wall, and limbs. ${ }^{6}$ Well known brain stem circuits are involved in the generation of the acoustically evoked startle reflex. ${ }^{7}$ Latencies of the activity in the orbicularis oculi muscles elicited by the acoustic startle stimulus are significantly shorter compared with the latencies of other muscle groups-for example, sternocleidomastoid muscles. ${ }^{8}$ Therefore, some authors suggest that the startle response of orbicularis oculi muscles is an acoustic blink reflex, which is separate from the startle reflex. However, other authors consider the acoustic blink reflex to be the first event of the startle reflex. $^{69}$

Brown et $a l^{10}$ first demonstrated that the amplitude of the acoustic startle reflex in rats can be augmented by presenting the eliciting acoustic startle stimulus together with a cue which has previously been paired with a painful shock. The paradigm is called "fear conditioned potentiation of the startle reflex". In this conditioning paradigm, a central state of fear has been proposed to serve as the conditioned response. In human studies, the conditioned fear is commonly determined by the increase of the acoustic blink reflex amplitude in the presence of a cue previously paired with an unpleasant stimulus. ${ }^{11-13}$

Stimulation of the anterior cerebellar vermis was found to augment the startle reflex in the fear potentiated startle paradigm in rats, ${ }^{14}$ suggesting involvement of the vermis in fear conditioning of the startle reflex in animals. However, Hitchcock and Davis ${ }^{15}$ transected the cerebellar peduncles bilaterally without any effect on the conditioned fear. To date, it remains open whether the cerebellar vermis is involved in fear conditioned potentiation of the startle reflex. The aim of the present study was to investigate a possible involvement of the medial cerebellum in fear conditioned potentiation of the acoustic blink reflex in humans. 
Table 1 Basic characteristics of patients with cerebellar lesions

\begin{tabular}{llllllll}
\hline Patient No & Age & Sex & $\begin{array}{l}\text { Aetiology of cerebellar } \\
\text { disorder }\end{array}$ & $\begin{array}{l}\text { Date of onset of } \\
\text { disorder/ operation }\end{array}$ & $\begin{array}{l}\text { Localisation of } \\
\text { cerebellar lesion }\end{array}$ & $\begin{array}{l}\text { Total WFN } \\
\text { ataxia score }\end{array}$ & $\begin{array}{l}\text { Extra-cerebellar } \\
\text { signs }\end{array}$ \\
\hline 1 & 29 & M & Medulloblastoma & 1996 & Midline & $28 / 100$ & None \\
2 & 27 & M & Astrocytoma & 1976 & Midline & $0 / 100$ & None \\
3 & 22 & M & Astrocytoma & 1992 & Midline & $6 / 100$ & None \\
4 & 11 & F & Astrocytoma & 1996 & Midline & $21 / 100$ & None \\
5 & 15 & M & Astrocytoma & 1992 & Midline & $28 / 100$ & None \\
6 & 19 & M & Astrocytoma & 1994 & Midline & $8 / 100$ & None \\
7 & 39 & M & IDCA & 1977 & Diffuse & $36 / 100$ & None \\
8 & 53 & M & SCA6 & 1984 & Diffuse & $41 / 100$ & None \\
9 & 58 & F & Haemangioblastoma & $1984+1991$ & Right cerebellar hemisphere & $3 / 100$ & None \\
10 & 64 & M & Haemorrhagic stroke & 1993 & Right cerebellar hemisphere & $2 / 100$ & None \\
\hline
\end{tabular}

$\mathrm{M}=$ Male; $\mathrm{F}=$ female; IDCA=idiopathic cerebellar ataxia; SCA=spinocerebellar ataxia; WFN=World Federation of Neurology; WFN ataxia score: maximum score $=100$, the higher the score the worse the clinical ataxia.

\section{Material and methods}

SUBJECTS

Ten cerebellar patients were tested (eight males, two females; mean age 33.8 (SD 18.8) years, range 11-64 years). Eight patients presented with lesions primarily of the medial cerebellum. Six of them had cerebellar lesions due to surgery for midline tumours such as astrocytoma $(n=5)$ or medulloblastoma $(n=1)$. The two other patients presented with degenerative disorders classified as idiopathic cerebellar ataxia ((IDCA) according to Harding ${ }^{16}$ ) in one patient and spinocerebellar ataxia type 6 (SCA 6) in the other. Both patients showed marked atrophy of the cerebellar vermis on recent MRI. In addition, two cerebellar patients presented with lateral lesions due to haemorrhagic stroke or surgery of haemangioblastoma. The degree of ataxia was assessed according to the International Cooperative Ataxia Rating Scale of the World Federation of Neurology (WFN) scale. ${ }^{17}$ None of the patients showed signs of extracerebellar lesions on brain scans and on neurological examination. Descriptive statistics of the patients are shown in the table.

Sixteen age and sex matched controls (13 male, three female; mean age 29.6 (16.1) years, range 12-64 years) without neurological or general medical limitations participated. There were no hearing or visual abnormalities, based on clinical examination in patients and controls. All subjects gave informed consent. The ethics committee of the University of Essen has approved the project.

\section{PROTOCOLS}

A fear conditioned potentiation paradigm of the startle reflex based on previous animal and human studies was used. ${ }^{10}{ }^{13}$ Subjects were in a supine position on an examination couch in a quiet room with their eyes closed. The protocol was divided into three phases. In the first, the patients and controls passed through a habituation phase, in which subjects received solely the acoustic blink stimulus. In the second, fear conditioning phase the subjects received paired presentations of a conditioned stimulus (CS), a light, and an electric shock as unconditioned fear stimulus (US). In the third, potentiation phase the subjects passed through a pseudorandom order of acoustic blink stimuli alone and acoustic blink stimuli paired with the CS. In all three phases the EMG of the orbicularis oculi muscles were recorded. Skin conductance and the heart rate were measured to obtain objective data about the subject's state of fear. Stimuli were presented with a pseudorandom interstimulus interval of 13-19 seconds (mean 16 seconds). The interval between phases was 5-10 seconds. After the potentiation phase, subjects were asked to mark their subjective state of fear and intensity of pain sensation during the experimental procedure, using visual scales.

\section{Phase 1: habituation}

Subjects received 20 successive acoustic blink stimuli (for parameters see "testing apparatus").

\section{Phase 2: fear conditioning}

Subjects received 20 successive pairs of the CS (light) and US (electric shock).

\section{Phase 3: potentiation}

Subjects received an initial refreshment of the habituation of the acoustic blink reflex with five successive acoustic blink stimuli. The subsequent potentiation protocol consisted of an alternating stimulus sequence of three different (pairs of) stimuli: (a) six pairs of CS (light) and US (electric shock), (b) 12 CS paired with a single tone burst, and (c) 12 single tone burst alone. These three (pairs of) stimuli were presented in a pseudorandom order, which was the same for all subjects. Six pairs of US and CS were presented to refresh the conditioning phase. Moreover, the subjects were less able to foresee the stimulus sequence. The following list shows the whole stimulus sequence of the potentiation protocol ( $\mathrm{T}$ ("tone")=single tone burst alone; LT ("light tone")=pair of the CS and the single tone burst; LS ("light shock")=pair of the US and CS): T-T-T-T-TLT-T-LS-T-LT-LT-T-LS-T-LT-LT-T-LS-T-LTT-LT-LS-LT-T-LT-T-LS-T-LT-T-LT-LS-LT-T.

\section{TESTING APPARATUS}

Acoustic blink stimulus and unconditioned and conditioned stimulus

The acoustic blink stimulus was a single tone burst of $80 \mathrm{~dB}$ with a duration of $50 \mathrm{~ms}$ presented bilaterally via stereophonic headphones. In the interval between two blink stimuli the subjects received a white noise of $70 \mathrm{~dB}$ to mask environmental noise. Shock stimuli (US) were applied via a bipolar stimulus electrode at the subject's right index finger with a duration of $100 \mathrm{~ms}$. The intensity of the electric shock was determined before the habituation phase by application of increasing 
shock stimuli until the individually highest tolerable shock intensity was reached (patients: mean (SD): 3.1 (1.5) mA; controls: mean (SD): 3.3 (1.2) $\mathrm{mA}$; no significant group difference: $\mathrm{p}=0.58(t$ test $))$.

The conditioned stimulus (CS) was a white light presented through blackened diving goggles with two small electric light bulbs of 30 W which were fastened inside at the top of the goggles. The light increased in its intensity in a ramp form over 2 seconds. The US coterminated with the CS.

\section{Acoustic blink reflex}

EMG recordings of the acoustic blink reflex were obtained in the habituation and potentiation phases. The EMG was recorded with bipolar surface electrodes placed bilaterally over the orbicularis oculi muscles (sampling rate $500 \mathrm{~Hz}$ ) from 2 seconds before onset and 1 second after ending of the acoustic stimulus. EMG signals were fed to EMG amplifiers (sensitivity $50 \mu \mathrm{V} /$ division), full wave rectified and filtered (lower frequency limits $5 \mathrm{~Hz}$, upper frequency limits $5 \mathrm{kHz}$ ) and further filtered off line $(100 \mathrm{~Hz})$. The latency of onset and end of the acoustic blink reflex were visually determined by placing a cursor at the earliest and the latest times that EMG activity in a single trial deviated from the baseline level. The peak amplitude of the acoustic blink reflex was determined within a fixed interval of 100 ms after onset. Baseline muscle activity for an interval of $100 \mathrm{~ms}$ was ascertained. The mean baseline muscle activity was subtracted from the peak amplitude of the acoustic blink reflex. In the habituation phase the peak amplitude of the blink reflex was normalised by assigning an arbitrary value of $100 \%$ to each subject's mean of all 20 trials. In the potentiation phase the peak amplitude of the blink reflex was normalised by assigning an arbitrary value of $100 \%$ to each subject's mean of peak amplitudes evoked by all 24 single tone and paired CS tone trials during the potentiation phase. There was no significant difference between the results of the subject's left and right side. Therefore the means of both sides are presented together.

\section{Heart rate and skin conductance}

Heart rate and skin conductance response were obtained in the fear conditioning phase. The heart rate was recorded via limb leads from 2 seconds before until 8 seconds after the CS-US pairs. Skin conductance was measured using two electrodes placed at the ball of the subject's left thumb and the ball of the fifth finger. The peak amplitude of the skin conductance response in the interval of 1 to 8 seconds after the end of the unconditioned stimulus (electric shock) was defined. Because of the short CS-US window, we were not able to investigate the first and second interval response of the skin conductance separately.

\section{Subjective state of fear and pain sensation}

After the end of the potentiation phase the subjects were asked to mark on a line with a length of $16 \mathrm{~cm}$ their state of fear and pain sensation, with the left end representing "no fear" and the right end "strongest fear you can imagine", respectively "no pain" and "strongest pain you can imagine". Furthermore, subjects were asked to name a number from 0 to 10 to describe their state of fear and intensity of pain sensation during the experiment with 0 representing "no fear" or "no pain", and 10 "strongest fear you can imagine" or "strongest pain you can imagine". All parameters were not significantly different between both groups (all $\mathrm{p}$ values $>0.2$ ( $t$ test)).

\section{Results}

The results for the eight patients with involvement of the medial cerebellum will be presented first. The results for the two patients with lateral cerebellar lesions will be presented separately at the end of this section.

\section{HABITUATION PHASE}

The recordings of the orbicularis oculi muscles showed a significant habituation effect within few trials in both the cerebellar and control groups (fig 1).

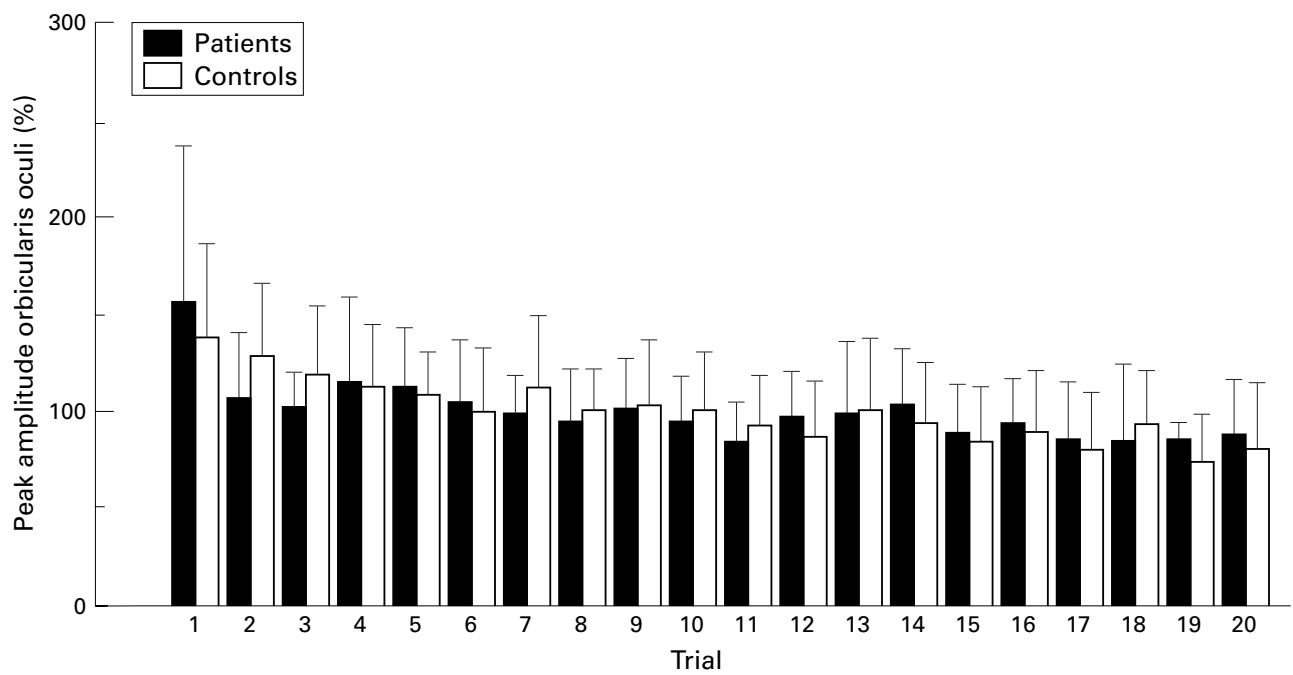

Figure 1 Habituation phase shows the mean (SD) of normalised peak amplitudes of the acoustic blink reflex during the habituation phase recorded from the orbicularis oculi muscles. Note the provable habituation effect with no significant difference between the patient and the control group. 

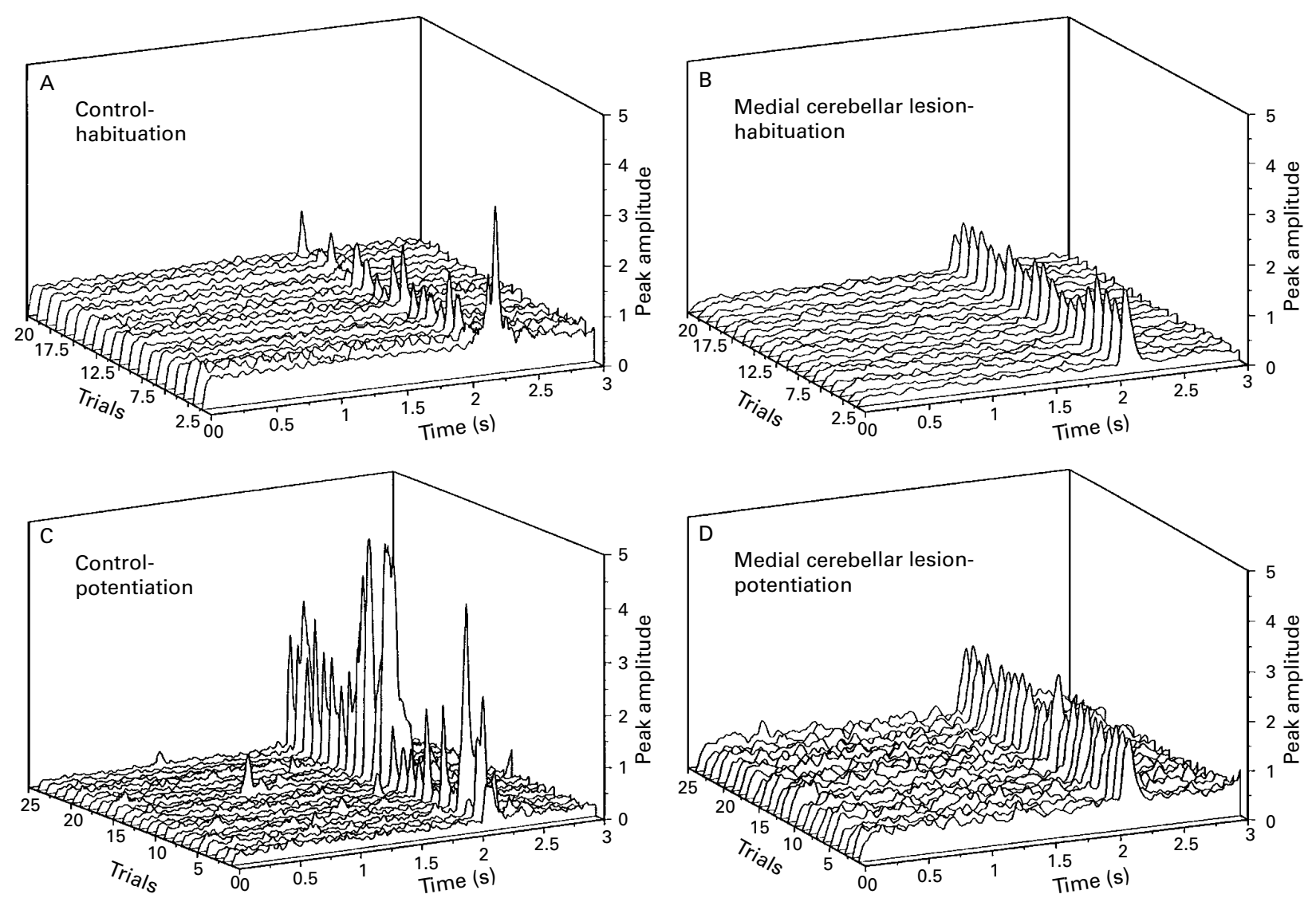

Figure 2 EMG of the orbicularis oculi muscles during the habituation $(A+B)$ and the potentiation $(C+D)$ phases in a control subject $(A+C)$ and a cerebellar patient $(B+D)$ (subject 6, table). The first 12 trials in the potentiation phase show blink reflexes evoked by the tone alone. The following 12 trials show the potentiated blink responses evoked by pairs of light and tone.

Characteristic examples of a control subject and a cerebellar patient are shown in figure $2 \mathrm{~A}$ and $\mathrm{B}$.

For group comparisons the normalised peak amplitudes of the acoustic blink reflex of successive blocks of four trials were averaged (=5 blocks). The univariate repeated measures analysis (MANOVA, group=between subject factor, block=within subject factor) disclosed a significant habituation effect of the peak amplitudes of the acoustic blink reflex (block effect $p<0.001$ ) and showed no significant differences between cerebellar patients and control subjects (group $\times$ block interaction: $\mathrm{p}=0.886)$.

FEAR CONDITIONING PHASE

In the fear conditioning phase the maximal amplitudes of the skin conductance response were significantly higher in the control group compared with the cerebellar group. In both groups the maximal amplitudes of the skin conductance response decreased within the 20 trials. Figure 3 shows the maximal skin conductance responses of both groups in five blocks of four trials. There was no significant difference of the mean or change of heart rate comparing the cerebellar and control groups during the fear conditioning phase.

For group comparisons heart rate and skin conductance response were averaged in blocks of four successive trials (=5 blocks). The univariate repeated measures analysis with maximal amplitudes of the skin conductance response as dependent variable showed a significant group $(\mathrm{p}=0.03)$ and a significant block effect $(\mathrm{p}=0.006)$ but no significant group $\times$ block interaction $(p=0.148)$. Post hoc testing showed a significant smaller mean maximal amplitude of the skin conductance response in the group of patients than in the control group ( $t$ test; $\mathrm{p}<0.01$ ). The multivariate analysis with mean heart rate and SD of the heart rate as dependent variables showed no group $(p=0.373)$, block $(p=0.216)$, or group $\times$ block effect $(p=0.446)$. There was no correlation between the results of the mean heart rate and the skin conductance response $(R=0.122$, Pearson's correlation coefficient; $\mathrm{p}=0.607)$.

\section{POTENTIATION PHASE}

The potentiation effect was determined as the difference in the normalised peak amplitude of the acoustic blink reflex evoked by the CS tone pairs and evoked by the tone alone trials.

The potentiation effect was significantly larger in control subjects than in the group of patients (fig 4). Fig $2 \mathrm{C}$ and D shows the significant increase in blink reflex amplitude in paired trials in one representative control subject, whereas no change in amplitude was seen in a patient with a midline cerebellar lesion (subject 6 in the table).

In controls, the peak amplitude of the acoustic blink reflex evoked by the single tone burst paired with the CS (mean (SD) 110.8 $(17.9) \%$ ) was larger than the peak amplitude 


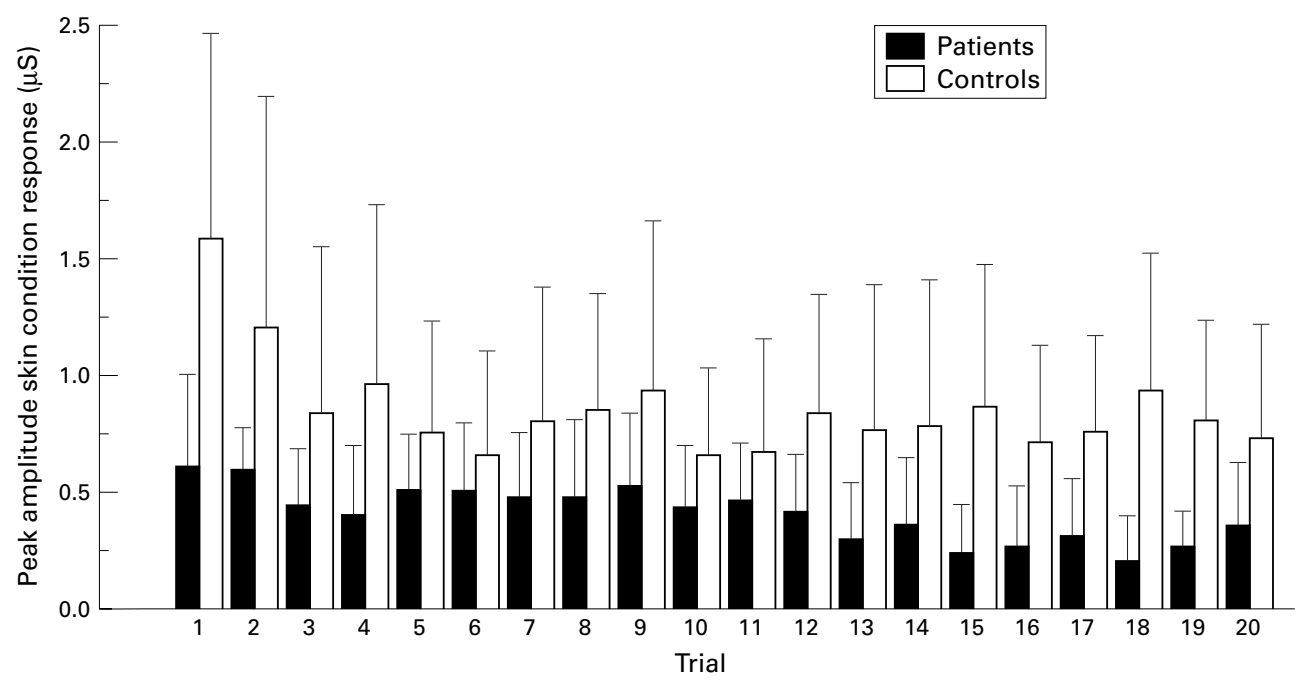

Figure 3 Fear conditioning phase: skin conductance response presents the mean (SD) of maximal amplitudes of the skin conductance response during the fear conditioning phase in the control and cerebellar group. The difference between the patient and the control group was significant $(p<0.001)$.

evoked by a single tone burst (mean (SD) 89.2 $(17.9) \%)$. The mean potentiation effect in controls was $21.6 \%$ (SD 35.6\%). The patient group showed a comparable blink reflex evoked by the single tone burst alone (mean (SD) peak amplitude $103.2(7.6) \%)$. By contrast with controls, the mean (SD) peak amplitude evoked by the single tone burst paired with the CS did not increase (96.8 (7.6)\%). The difference between the mean peak amplitudes evoked by the two different kinds of stimuli was $-6.4 \%$ (SD 15.3\%) in the cerebellar group.

A univariate analysis (group=between subject factor, condition (tone alone trials $v$ CS-tone pairs) =within subject factors) with normalised peak amplitudes as dependent variables was performed. This analysis showed a statistically significant group effect $(p<0.001)$ and interaction of group and condition $(p=0.048)$, which reflected the higher potentia- tion effect in the control group as compared to the cerebellar group. There was no statistically significant condition effect $(\mathrm{p}>0.2)$. Omission of the two patients with degenerative disease showed similar results- that is, post hoc analysis showed a significant reduced potentiation effect in patients with medial surgical lesions compared to the controls $(\mathrm{p}=0.026, t$ test $)$.

RESULTS OF PATIENTS WITH LATERAL

CEREBELLAR LESIONS

The two patients with lateral cerebellar lesions (subjects 9, 10; table) showed no disturbances of the acoustic blink reflex habituation. Shock intensity, skin conductance response, and heart rate were not significantly different from the controls.

Both patients with lateral cerebellar lesions showed provable potentiation effects during the potentiation phase which were in the range

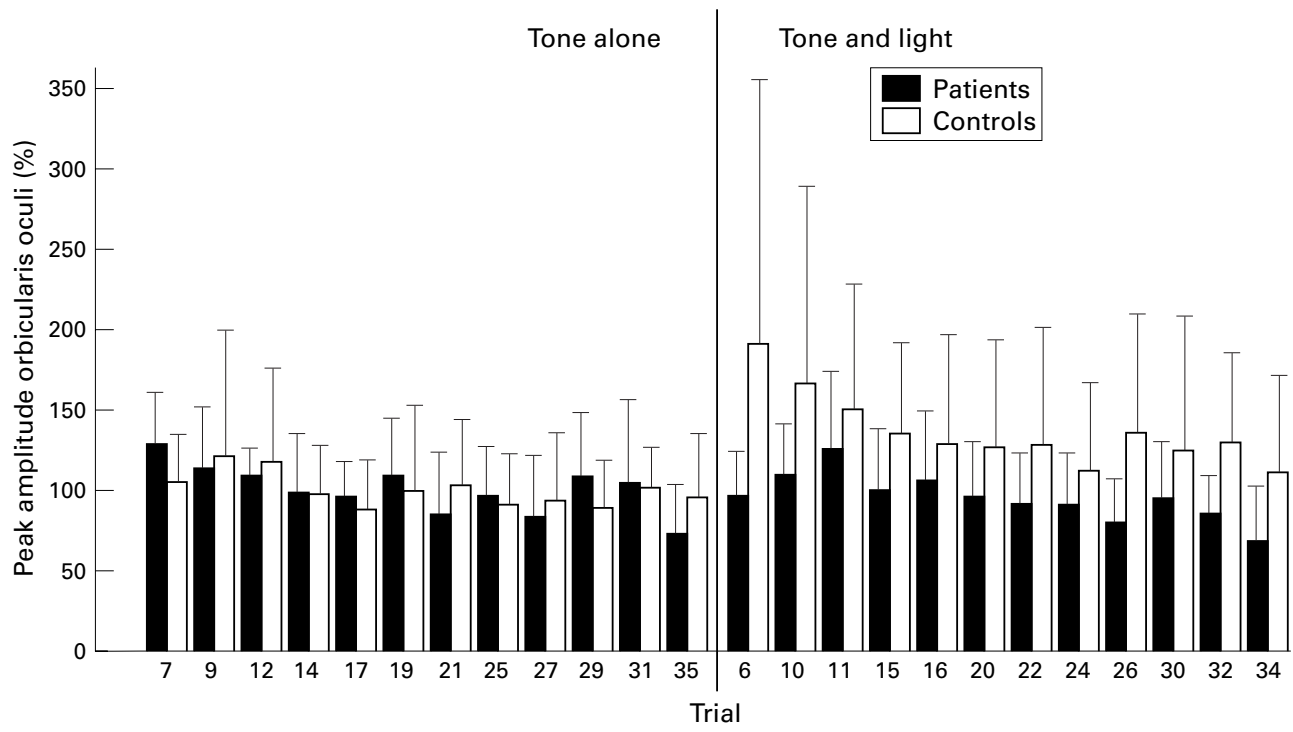

Figure 4 Potentiation phase illustrates the mean (SD) peak amplitudes recorded at the orbicularis oculi muscles of the patient group with medial or diffuse cerebellar lesions and the control group. The first 12 trials denote the peak amplitudes evoked by the single tone burst alone. The subsequent 12 trials demonstrate the peak amplitudes evoked by the single tone bursts paired with the CS (light). Note the increase in the control subject's mean peak amplitude evoked by the single tone burst paired with the light, but not in the cerebellar subjects. 
of the control subjects. Patient 9 showed a mean normalised peak amplitude evoked by the single tone burst alone of $93.6 \%$ and evoked by the single tone burst paired with the CS of $106.4 \%$. Patient 10 had a mean normalised peak amplitude evoked by the single tone burst of $82.6 \%$ and evoked by the single tone burst paired with the CS of $117.4 \%$. Thus, the potentiation effect was $12.8 \%$ for patient 9 and $34.8 \%$ for patient 10 and therefore within normal limits.

\section{Discussion}

The results of the three phases of fear conditioned potentiation of the acoustic blink reflex - that is, habituation, fear conditioning, and potentiation - will be discussed separately. Unless otherwise stated, the patient group refers to the group of patients with lesions involving the medial cerebellum. The findings in the two patients with lateral cerebellar lesions will be discussed in a separate paragraph at the end of this section.

HABITUATION OF THE ACOUSTIC BLINK REFLEX Our results showed a significant habituation of the acoustic blink reflex within 20 trials in both the control group and the patient group. The findings of preserved habituation effects in patients with medial cerebellar lesions are supported by animal studies, which showed that short term habituation of the startle reflex depends critically on the brain stem, but not on the cerebellum. ${ }^{35}$

At first sight contradictory, a previous PET study of our group demonstrated a significant decrease of cerebral blood flow in medial parts of the cerebellum during ongoing habituation of the startle reflex in healthy subjects. ${ }^{18}$ However, the interstimulus interval was significantly longer in our PET study compared with the present study (10 minutes $v$ 13-19 seconds). Previous animal studies showed that lesions of the vermis eliminate long term habituation of the startle reflex, but not short term habituation..$^{3-5}$ Therefore, the change of vermal blood flow in our PET study might well reflect a form of long term habituation.

In summary, the present findings suggest that the medial cerebellum is not essentially involved in short term habituation of the acoustic blink reflex in humans.

FEAR CONDITIONING

The heart rate and skin conductance response were measured to obtain objective data representing the state of fear during the fear conditioning phase.

The mean values of the maximal amplitude of the skin conductance response were significantly lower in the patient group than in the control group. It should be emphasised that the lower amplitude in patients did not correlate with a lower state of subjective fear or shock intensity. Therefore, the lower amplitude of skin conductance responses in cerebellar patients may suggest a possible involvement of the cerebellum in autonomic functions. Interestingly, there is increasing evidence of nonmotor functions of the human cerebellum such as in certain cognitive tasks, behaviour, and attention, including anecdotal reports of autonomic dysfunction in patients with vermal cerebellar lesions..$^{19} 20$

However, inspection of the time course of changes in amplitude of the skin conductance response during the conditioning phase disclosed no difference between control subjects and cerebellar patients. These results agree with those of Daum et $a l^{1}$ findings of intact electrodermal conditioning in an eyeblink conditioning paradigm in cerebellar patients. Both the present negative findings and those of Daum et $a l,{ }^{21}$ however, must be interpreted with caution. Daum et $a l^{1}$ pointed out that because eyeblink conditioning requires a CS-US interval shorter than 1 second, and given the long and variable unconditioned skin conductance onsets, their study could not assess CR s and UR s independently. Although the CS-US interval used in the present study was significantly longer (2 seconds) CRs and URs were indistinguishable in most cases, and therefore the peak amplitude of the entire response was assessed. Furthermore, both groups showed a decline of the peak amplitudes of the skin conductance response within the conditioning phase. The decline of the peak amplitudes may reflect habituation rather than conditioning during the fear conditioning phase.

Neither the subjective data of fear nor the results of mean heart rate and changes of heart rate during the fear conditioning phase showed significant differences between the control and patient groups. However, our findings did not show significant conditioning effects of the heart rate in both the control and cerebellar groups. Fear conditioned bradycardia has been shown in healthy subjects and intact animals ${ }^{22} 23$ and studies in animals with midline cerebellar lesions disclosed disturbed fear conditioned bradycardia. ${ }^{24}{ }^{25}$ Similarly to electrodermal conditioning, it cannot be decided by the present study whether or not conditioning of the heart rate is disturbed by cerebellar lesions because the CS-US time window was most likely too short to show heart rate deceleration or acceleration in controls. For example, the CS-US interval found to be optimal for heart rate conditioning was 4.0 seconds in animal studies. ${ }^{26}$

In summary, the lower amplitude of the skin conductance response in cerebellar patients may indicate that fear conditioning is impaired in patients with medial cerebellar lesions. However, further studies with sufficiently long CS-US time windows are needed to clarify if the human cerebellum is involved in fear (that is, skin conductance and heart rate) conditioning.

FEAR CONDITIONED POTENTIATION OF THE ACOUSTIC BLINK REFLEX

In control subjects the mean peak amplitudes of the blink reflex evoked by pairs of the fear conditioned stimulus (the light) and the single tone burst was larger than the mean peak amplitudes evoked by the acoustic blink stimulus alone. The increase of the mean peak amplitude evoked by presenting the acoustic 
blink stimulus together with the fear conditioned stimulus was significantly lower in the patient group compared with the controls. These results suggest an involvement of the human cerebellum in the fear conditioned potentiation of the acoustic blink reflex.

Previous studies looking for a possible role of the cerebellum in fear conditioned potentiation are sparse and contradictory. Albert et $a l^{14}$ showed that electric stimulation of the anterior cerebellar vermis augmented the potentiation effect in rats. By contrast, Hitchcock and Davis $^{15}$ transected the cerebellar peduncles without blocking the potentiated startle effect in rats. These findings suggest that the cerebellum may be involved, but may not be essential, in this learning paradigm.

Fear conditioned potentiation may be impaired by medial cerebellar lesions because of a disturbance in the fear conditioning or the potentiation itself, or both. As mentioned above, the mean amplitude of the maximal skin conductance response was reduced in the patient group. The amplitudes of the skin conductance response are related to the affective valence of a stimulus. ${ }^{23}$ Therefore the significant reduction in patients with medial cerebellar lesions may reflect a disturbance in the conditioning of fear. In fact, correlation analysis shows a clear trend of an influence of the magnitude of the skin conductance response on the potentiation effect $(R=0.425, \mathrm{p}=0.06)$.

In conclusion, medial lesions of the human cerebellum affected fear conditioned potentiation of the acoustic blink reflex. On the basis of the present findings, however, it cannot be decided if the potentiation process itself or/and the fear conditioning are impaired.

RESULTS IN PATIENTS WITH LATERAL CEREBELLAR LESIONS

Two patients with circumscribed lesions of the cerebellar hemispheres were tested. In both patients, habituation, fear conditioning, and fear conditioned potentiation of the acoustic blink reflex were preserved. Although these findings should be confirmed in a larger patient population, the present results of unimpaired fear conditioned potentiation in patients with lateral lesions suggest a role of medial cerebellar parts in this associative learning paradigm.

The present findings are further supported by previous animal and human studies, which showed a functional compartmentalisation of the cerebellum for conditioning of non-specific and specific aversive reactions. The intermediate cerebellum is involved in classical conditioning of specific aversive reactions, in particular of the eyeblink and limb flexion reflex, ${ }^{12}$ whereas, as mentioned above, the conditioning of the heart rate, a non-specific aversive reaction, is disturbed by lesions of the vermal part of the cerebellum in rats. ${ }^{25}$ Likewise, recent PET studies of our group demonstrated involvement of the intermediate cerebellum in limb flexion conditioning and of more medial parts of the cerebellum in a habituation paradigm of the startle reflex. ${ }^{18} 27$ The present findings emphasise a role of the medial cerebellum for associative learning of nonspecific aversive reactions in humans.

The study was supported by a grant from the Deutsche Forschungsgemeinschaft to DT (DFG Ti 239/2-1, Ti 239/2-2) and by a grant from the Wilhelm-Sander-Stiftung to FPK (AZ 904090/ 2). We thank $H$ G Elles for technical assistance and F Schaefer for providing us with the preamplifier for skin conductance recordings.

1 Bloedel JR, Bracha V. On the cerebellum, cutaneomuscular reflexes, movement control and the elusive engrams of memory. Behav Brain Res 1996;68:1-44.

2 Thompson RF, Krupa DJ. Organization of memory traces in the mammalian brain. Anmu Rev Neurosci 1994;17:51949 .

3 Leaton RN, Supple WF. Cerebellar vermis: essential for long-term habituation of the acousitic startle response. Science 1986;232:513-15.

4 Lopiano L, De'Sperati C, Montarolo PG. Long-term habituation of the acoustic startle response: role of the cerebellar vermis. Neurosci 1990;35:79-84.

5 Leaton RN, Supple WF. Medial cerebellum and long-term habituation of acoustic startle in rats. Behav Neurosci 1991; 105:804-16.

6 Landis C, Hunt WA. The startle pattern. New York: Farrar and Rinehart, 1939.

7 Yeomans JS, Frankland PW. The acoustic startle reflex: neurons and connections. Brain Res Rev 1996;21:301-14.

8 Brown P, Rothwell JC, Thompson PD, et al. New observations on the normal auditory startle reflex in man Brain 1991;114:1891-902.

9 Wilkins DE, Hallett M, Wess MM. Audiogenic startle reflex of man and its relationship to brain syndromes: a review. Brain 1986;109:561-73.

10 Brown JS, Kalish HI, Farber IE. Conditioned fear as revealed by magnitude of startle response to an auditory stimulus. F Exp Psychol 1951;41:317-28.

11 Ross LE. Conditioned fear as a function of CS-UCS and probe stimulus intervals. F Exp Psychol 1961;61:265-73.

12 Hamm AO, Greenwald MK, Bradley MM, et al. Emotional learning, hedonic change, and the startle probe. $\mathcal{F}$ Abnorm Psychol 1993;102:453-65.

13 Lipp OV, Sheridan J, Siddle DAT. Human blink startle during aversive and nonaversive pavlovian conditioning. $\mathcal{F}$ Exp ing aversive and nonaversive pavlovian con
Psychol Anim Behav Process 1994;20:380-9.

14 Albert TJ, Dempsey CW, Sorenson CA. Anterior vermal stimulation: effect on behavior and basal forebrain neurochemistry in rat. Biol Psychiatry 1985;20:1267-76.

15 Hitchcock JM, Davis M. Lesions of the amygdala, but not of the cerebellum or red nucleus, block conditioned fear as measured with the potentiated startle paradigm. Behav Neurosci 1986;100:11-22.

16 Harding AE. Clinical features and classification of inherited ataxias. Adv Neurol 1993;61:1-14.

17 Trouillas $\mathrm{P}$, Takayanagi $\mathrm{T}$, Hallett $\mathrm{M}$, et al. International cooperative ataxia rating score for pharmacological assessment of the cerebellar syndrome. F Neurol Sci 1997; 45:20511.

18 Timmann D, Musso C, Kolb FP, et al. Involvement of the human cerebellum during habituation of the acoustic startle response: a PET study. $\mathcal{F}$ Neurol Neurosurg Psychiatry 1998;65:771-3.

19 Berquin PC, Giedd JN, Jacobsen LK, et al. Cerebellum in attention-deficit hyperactivity disorder: a morphometric MRI study. Neurology 1998;50:1087-93.

20 Schmahmann JD, Sherman JC. The cerebellar cognitive ffective syndrome. Brain 1998;121:561-79.

21 Daum I, Schugens MM, Ackermann H, et al. Classical conditioning after cerebellar lesions in humans. Behav Neurosci 1993;107:748-56.

22 Powell DA. Rapid associative learning: conditioned bradycardia and its central nervous system substrates. Int Phys Beh Sci 1994;29:109-33.

23 Lang PJ, Greenwald MK, Bradley MM, et al. Looking at pictures: affective, facial, visceral, and behavioral changes. Psychophysiol 1993;30:261-73.

24 Lavond DG, Lincoln JS, McCormick DA, et al. Effect of bilateral lesion of the dentate and interpositus cerebellar nuclei on conditioning of heart rate and nictitating membrane/eyelid responses in the rabbit. Brain Res 1984;305:323-30.

25 Supple WF, Leaton RN. Cerebellar vermis: essential for classical conditioned bradycardia in the rat. Brain Res 1990;509:17-23.

26 Powell DA, Lipkin M, Milligan ML. Concomitant heart rate and corneoretinal potential conditioning in the rabbit (Oryctolagus cuniculus): effects of caudate lesions. Learning and Motivation 1974;5:532-47.

27 Timmann D, Kolb FP, Baier C, et al. Cerebellar activation during classical conditioning of the human flexion reflex using positron emission tomography (PET). Neuroreport 1996;7:2056-60. 